\title{
BMJ Open Awareness and bispectral index (BIS) monitoring in mechanically ventilated patients in the emergency department and intensive care unit: a systematic review protocol
}

\author{
Ryan D Pappal, ${ }^{1}$ Brian W Roberts (D) , ${ }^{2}$ Winston Winkler, ${ }^{1}$ Lauren H Yaegar, ${ }^{3}$ \\ Robert J Stephens (D) , ${ }^{4}$ Brian M Fuller (D) ${ }^{5}$
}

To cite: Pappal RD, Roberts BW, Winkler W, et al. Awareness and bispectral index (BIS) monitoring in mechanically ventilated patients in the emergency department and intensive care unit: a systematic review protocol. BMJ Open 2020;10:e034673. doi:10.1136/ bmjopen-2019-034673

- Prepublication history and additional material for this paper are available online. To view these files, please visit the journal online (http://dx.doi. org/10.1136/bmjopen-2019034673).

Received 02 October 2019 Revised 11 February 2020 Accepted 12 February 2020

Check for updates

(C) Author(s) (or their employer(s)) 2020. Re-use permitted under CC BY-NC. No commercial re-use. See rights and permissions. Published by BMJ.

For numbered affiliations see end of article.

Correspondence to

Dr Brian M Fuller;

fullerb@wusm.wustl.edu

\section{ABSTRACT}

Introduction Accidental awareness with recall is one of the most feared complications for patients undergoing general anaesthesia and can lead to post-traumatic stress disorder in up to $70 \%$ of patients experiencing it. To reduce the incidence of awareness with recall, the bispectral index monitor is recommended for patients receiving total intravenous anaesthetics, especially those receiving neuromuscular blockers. While extensive investigation into awareness and bispectral index monitoring has occurred for operating room patients, this has not extended to other clinical arenas where sedated and mechanically ventilated patients are cared for, namely the intensive care unit and emergency department. The purpose of this systematic review is to assess the world's literature to determine the incidence of awareness with paralysis in mechanically ventilated patients and the impact of bispectral index monitoring for reducing this complication.

Methods and analysis Randomised trials and nonrandomised studies are eligible for inclusion. With aid from a medical librarian, an electronic search will include Ovid Medline, Embase.com, Scopus, Cochrane Database of Systematic Reviews and Cochrane Central Register of Controlled Trials. To find data published in abstract form, literature from professional society conferences (2010-2019) will be manually searched. Two authors will independently review search results and consensus will be reached with assistance from a third author, as needed. Heterogeneity and publication bias will be assessed and reported. If possible and appropriate, a meta-analysis of the data will be conducted for quantitative data analysis.

Ethics and dissemination The proposed systematic review does not require ethical approval, as it is conducted at the study level and does not involve individual patientlevel data. Results will be disseminated by data sharing via academically established means, presentation at local and national scientific meetings and publication as a peerreviewed manuscript.

PROSPERO registration number The protocol has been submitted to International Prospective Register of Systematic Reviews and is awaiting registration.
Strengths and limitations of this study

- This work will be the most comprehensive assessment of awareness with paralysis in mechanically ventilated, sedated, critically ill patients to date.

- The results are expected to shed light on the incidence of a highly morbid complication, identify scientific gaps in the literature and identify future research targets to improve postintubation care.

- Relevant studies are unlikely to be missed, given the methodological rigour of the systematic review.

- Meaningful results could be limited if there is a paucity of literature regarding awareness with paralysis in patients in the emergency department and intensive care unit.

\section{INTRODUCTION}

Accidental awareness with recall (AWR) is one of the most feared complications for patients undergoing general anaesthesia in the operating room (OR). An incidence of 1-2 cases /1000 is supported by data from singlecentre and multicentre cohort studies, as well as a randomised controlled trial. ${ }^{1-3}$ This topic is germane in the care of OR patients, as multiple studies have shown an association between accidental awareness and posttraumatic stress disorder (PTSD), which can be crippling and refractory to treatment. ${ }^{4-8}$ Risk factors for AWR include: (1) underdosing of anaesthesia; (2) lack of protocolled monitoring of sedation depth; (3) the use of neuromuscular blocking drugs (ie, paralytics) and (4) a total intravenous anaesthetic approach (vs inhaled anaesthetics).

The bispectral index monitor (BIS) is a processed electroencephalogram (EEG) device applied non-invasively to the forehead via an adhesive electrode sensor strip. The BIS index provided as the output from the 
device is a dimensionless number ranging from 0 , indicating suppression of detectable brain activity, to 100 , indicating the awake state. A BIS of $<60$ has a high sensitivity for identifying drug-induced unconsciousness, and maintaining BIS values between 40 and 60 has been the clinical strategy investigated for reducing the incidence of AWR in the OR population. ${ }^{1-11}$ Extensive investigation into the role of BIS monitoring for decreasing the chance of AWR has been conducted in the OR. While not superior to a protocolled monitoring strategy based on alarms which monitor end-tidal anaesthetic concentration, BIS is superior to clinical signs alone, routine clinical care (ie, non-protocol driven monitoring) and for patients receiving total intravenous anaesthesia. ${ }^{19-13}$ Therefore, evidence-based recommendations regarding mechanically ventilated patients in the OR endorse objective brain monitoring using BIS as a means to reduce the incidence of AWR in patients receiving total intravenous anaesthetics, especially in those receiving neuromuscular blockers. $^{1214}$

This focus on awareness and BIS monitoring has yet to extend to other clinical arenas where sedated and mechanically ventilated patients are cared for, namely the intensive care unit (ICU) and emergency department (ED), where hundreds of thousands of patients require mechanical ventilation annually. ${ }^{15} 16$ There is rationale to believe that the incidence of awareness with paralysis could be higher in these areas when compared with the OR, given the vastly different practice and staffing models that exist (ie, nursing/clinician staffing ratios, more hectic environment, less monitoring). Case reports and small cohort studies have documented an incidence of awareness with paralysis in patients in ICU as high as $35 \% .{ }^{17} 18$ With respect to BIS monitoring, most ICU studies have assessed the correlation between clinical sedation scales and BIS index values. ${ }^{19}$ The data regarding awareness with paralysis are quite limited, though one study did show a reduction in awareness with paralysis from $18 \%$ to $4 \%$ with the use of a BIS monitor to guide sedation titration. ${ }^{17}$ Data from the ED domain are even more limited. Four small studies ( $\mathrm{n}=123$ patients total) showed an incidence of awareness with paralysis ranging from $5.9 \%$ to $50 \%,{ }^{20-23}$ but this was restricted to the endotracheal intubation procedure only. One small cohort study showed no association between BIS values and recall of endotracheal intubation in the ED. ${ }^{21}$

Given the negative impact that awareness with paralysis has on patient-centred outcomes, along with the volume of patients requiring mechanical ventilation in the ED and ICU domains, quantifying the incidence in this cohort is important. ${ }^{24}$ There are no systematic reviews on awareness with paralysis and BIS monitoring in mechanically ventilated patients in ED and ICU. An important next step in investigating this outside of the OR is to collate and analyse the existing world literature. In this systematic review, we aim to: (1) describe the global biomedical literature regarding awareness with paralysis and BIS monitoring in mechanically ventilated patients in ED and
ICU; (2) quantify the incidence of awareness with paralysis in this cohort of critically ill patients and (3) assess the impact of BIS monitoring in reducing awareness events. We hypothesise that: (1) the literature will be limited in terms of methodological rigour and quantity; (2) the incidence of awareness with paralysis will be higher than that reported in the OR and (3) BIS monitoring will be associated with a reduction in awareness events. By rigorously examining awareness and BIS monitoring in critically ill patients in ED and ICU, this systematic review will be an important contribution in the effort to provide goaloriented sedation in the postintubation period.

\section{METHODS AND ANALYSIS}

\section{Protocol and registration}

The Preferred Reporting Items for Systematic Reviews and Meta-Analysis Protocols (PRISMA-P) statement (see online supplementary material 1 ) has been used to prepare this systematic review protocol. The PRISMA statement and the Meta-analysis of Observational Studies in Epidemiology guidelines will be used to report the final results. If there is deviation from the protocol during the conduct of this systematic review, it will be reported with the final results, as well as the rationale for protocol deviation. The electronic search for the systematic review was conducted in June 2019; the intended completion date for the entire work is estimated to be in December 2019. This systematic review has been submitted to the International Prospective Register of Systematic Reviews and is awaiting registration.

\section{Search for and identification of studies}

An electronic search was designed in collaboration with a medical librarian experienced in the conduct of comprehensive systematic reviews, and she performed the electronic search. This search was designed to include the following databases: Ovid Medline, Embase.com, Scopus, Cochrane Database of Systematic Reviews and the Cochrane Central Register of Controlled Trials. The search terms and search strategy were established using a combination of standardised terms and keywords. The fully reproducible search strategy is provided in online supplementary material 2.

To identify additional studies, a manual screen of the references from the publications selected for inclusion will be conducted. To to find potentially unpublished data, we will manually search abstracts from the following meetings (2010-2019): American College of Emergency Physicians, Society for Academic Emergency Medicine, American Thoracic Society, CHEST, Society of Critical Care Medicine, European Society of Intensive Care Medicine, International Symposium on Intensive Care and Emergency Medicine, American Society of Anesthesiologists, European Society of Anaesthesiology, International Anesthesia Research Society and the American Association of Critical Care Nurses. To identify completed but not yet published studies, an online search of clinical 
trials registration (ClinicalTrials.gov) will be conducted. Authors of published and unpublished studies will be contacted as needed to clarify study details or data related to possible inclusion in the systematic review.

\section{Eligibility criteria}

Studies will include adult patients receiving mechanical ventilation in the ED, ICU or prehospital domains, and will have no language restriction. To provide the most comprehensive assessment of the existing literature, all publication types will be considered, provided there is some report of the outcomes of interest. Manuscripts that are reviews, correspondences, editorials or non-human studies will not be included.

There is no intervention with respect to the primary outcome of interest, which is largely a descriptive assessment of the incidence of awareness with paralysis in critically ill mechanically ventilated patients. In studies assessing the use of BIS monitoring, we will report the impact of BIS monitoring on the incidence of awareness with paralysis (comparison-BIS monitoring vs no BIS monitoring). The primary outcome of interest is the incidence of awareness with paralysis. We will also report on recall/memory of events. The secondary outcomes of interest are the reporting methods used to assess for awareness and how awareness was defined in each study, as well as studies reporting the use of BIS monitoring in mechanically ventilated patients. Descriptive reporting will also include the use of sedatives and neuromuscular blockers, if reported. We will also attempt to report outcome differences that exist between patients with and without awareness, including PTSD, delirium and lengths of stay (ventilator, ICU, hospital).

\section{Study selection and data abstraction}

A written protocol will be followed for the conduct of this systematic review (see online supplementary material 3). After identification of studies with the electronic search, two independent reviewers will screen the titles and abstracts for eligibility. On completion of this relevance screen, the included studies from the two reviewers will be compared with assess studies where disagreement exists. A third reviewer will assist in adjudicating study eligibility in cases of disagreement to reach consensus. Full-text articles will then be obtained and comprehensively reviewed for inclusion.

After this comprehensive review, data will be extracted from the included manuscripts using standardised data collection forms and collated into table format. The following data on study characteristics will be collected and placed in a table: author, year of publication, study design, number of patients included, characteristics of the patient population, awareness and BIS data, study quality, risk of bias and outcomes.

\section{Assessment of study quality}

The Cochrane Collaboration's tool for assessing the risk of bias in clinical trials will be used to assess quality of randomised clinical trials. We will report a summary assessment for the risk of bias for each studied outcome. The Newcastle Ottawa Scale will be used to assess quality for studies of observational design. Five or fewer points will indicate a high risk of bias (maximum of nine points).

\section{Assessment of publication bias}

A graphical display (funnel plot) of the size of the treatment effect against the precision of the trial will be used to evaluate for potential publication bias.

\section{Strategy for data synthesis}

After data are collated, descriptive data will be provided for data points such as: study characteristics, study quality, awareness reporting, BIS monitoring and medications used for sedation. This qualitative reporting will focus on data and outcomes related to awareness and BIS monitoring and including a comprehensive narrative overview of the included studies. If objective data analysis is possible (as opposed to qualitative reporting only), we will use a meta-analytic approach to quantitatively analyse the data. Factors determining whether meta-analysis is appropriate will be: clinical characteristics and heterogeneity of the studies, clarity of outcomes reporting, study design(s) and presence of bias. A random-effects model will be used to calculate pooled effect sizes and corresponding 95\% CIs between groups (eg, BIS monitor vs no BIS monitor). ORs will be calculated for binary data. Continuous outcomes will be reported as mean difference, and overall effect estimates will be generated using a $\mathrm{Z}$ test and presented as mean differences. A p value of $<0.05$ will be considered statistically significant.

The $\mathrm{I}^{2}$ statistic will be used to assess heterogeneity between studies. We will perform sensitivity and subgroup analyses if the systematic review suggests that this is feasible and warranted to explore heterogeneity between studies. These will be reported as post hoc analyses, as there is currently no a priori identified subgroups of interest.

\section{Patient and public involvement}

No patients nor the public were involved in the development of the research question or study design and will not be involved in recruitment or conduct of the study.

\section{ETHICS AND DISSEMINATION}

There are no patient-level data to be analysed as part of this systematic review; it will be conducted at the study level. As such, no ethical approval will be required. Results from this work will be reported transparently, adhere to guideline methodology, be presented locally and at national scientific meetings and be published as a peer-reviewed manuscript.

This systematic review will be the most comprehensive assessment of awareness with paralysis and BIS monitoring in critically ill mechanically ventilated patients to date. Randomised trials, as well as non-randomised studies 
(prospective and retrospective cohort analyses, crosssectional studies, before-after trials) will be included. Inclusion of non-randomised studies is based on the following reasons: (1) the question of interest may not be investigated strictly with randomised trials, secondary to a lack of existing trial data; (2) to provide an explicit evaluation of strengths and weaknesses of the current literature; (3) to assess evidence of effects (benefit and harm) and (4) to provide evidence for the undertaking of further trials. We have therefore purposefully chosen this approach to provide the most comprehensive systematic review possible.

This study will refine our understanding of awareness with paralysis and BIS monitoring in the non-OR population of critically ill mechanically ventilated patients in the ED and ICU. We expect to demonstrate that awareness has been understudied in this patient population, yet is a significant complication for an important minority of patients. In that regard, we anticipate that this work will improve postintubation care and provide a platform for further investigation.

A possible limitation of this systematic review would be a lack of awareness and BIS monitoring data in the existing literature, which would limit any conclusions drawn, as well as our ability to meta-analyse the data. We will reduce this risk with our robust and comprehensive approach. However, if this is the case, a lack of data in this domain will provide valuable information to support further work. This systematic review will also focus only on BIS monitoring and not include other processed EEG monitors, limiting any conclusions regarding other processed EEG strategies that have been used to reduce the incidence of awareness with paralysis.

In conclusion, this systematic review will characterise and quantify the existing world literature regarding awareness with paralysis and BIS monitoring in mechanically ventilated patients. We anticipate that this study will yield additional information to guide postintubation practice for critically ill patients and generate hypotheses for future studies.

\section{Author affiliations \\ ${ }^{1}$ Washington University in Saint Louis School of Medicine, Saint Louis, Missouri, USA \\ ${ }^{2}$ Emergency Medicine, Cooper Medical School of Rowan University, Camden, New Jersey, USA \\ ${ }^{3}$ Bernard Becker Medical Library, Washington University in Saint Louis School of Medicine, Saint Louis, Missouri, USA \\ ${ }^{4}$ Emergency Medicine, Washington University in Saint Louis School of Medicine, Saint Louis, Missouri, USA \\ ${ }^{5}$ Emergency Medicine and Anesthesiology, Washington University, Saint Louis, Missouri, USA}

\section{Twitter Robert J Stephens @StephensRobJ}

Contributors RDP: conception and design of the work; acquisition, analysis and interpretation of data; substantial revisions to the manuscript. BWR: analysis and interpretation of data; design of the work; substantial revisions to the manuscript. WW: acquisition, analysis and interpretation of data; substantial revisions to the manuscript. LHY: design of the work; acquisition of data; substantial revisions to the manuscript. RJS: acquisition, analysis and interpretation of data; substantial revisions to the manuscript. BMF: conception and design of the work; acquisition, analysis and interpretation of data; drafting and substantial revisions to the manuscript; guarantor of the manuscript

Funding RDP received funding from the National Center For Advancing Translational Sciences of the National Institutes of Health under Award Number TL1TR002344. PI: Jay F Piccirillo, MD, FACS. Project Title: Washington University Institute of Clinical and Translational Sciences. The content of this publication is solely the responsibility of the authors and does not necessarily represent the official views of the National Institutes of Health.

Competing interests None declared.

Patient consent for publication Not required.

Provenance and peer review Not commissioned; externally peer reviewed.

Open access This is an open access article distributed in accordance with the Creative Commons Attribution Non Commercial (CC BY-NC 4.0) license, which permits others to distribute, remix, adapt, build upon this work non-commercially, and license their derivative works on different terms, provided the original work is properly cited, appropriate credit is given, any changes made indicated, and the use is non-commercial. See: http://creativecommons.org/licenses/by-nc/4.0/.

ORCID iDs

Brian W Roberts http://orcid.org/0000-0002-7690-997X

Robert J Stephens http://orcid.org/0000-0003-4660-251X

Brian M Fuller http://orcid.org/0000-0003-3757-756X

\section{REFERENCES}

1 Mashour GA, Shanks A, Tremper KK, et al. Prevention of intraoperative awareness with explicit recall in an unselected surgical population: a randomized comparative effectiveness trial. Anesthesiology 2012;117:717-25.

2 Sandin RH, Enlund G, Samuelsson P, et al. Awareness during anaesthesia: a prospective case study. Lancet 2000;355:707-11.

3 Sebel PS, Bowdle TA, Ghoneim MM, et al. The incidence of awareness during anesthesia: a multicenter United States study. Anesth Analg 2004;99:833-9.

4 Leslie K, Chan MTV, Myles PS, et al. Posttraumatic stress disorder in aware patients from the B-aware trial. Anesth Analg 2010;110:823-8.

5 Meyer BC, Blacher RS. A traumatic neurotic reaction induced by succinylcholine chloride. N Y State J Med 1961;61:1255-61.

6 Lennmarken C, Bildfors K, Enlund G, et al. Victims of awareness. Acta Anaesthesiol Scand 2002;46:229-31.

7 Whitlock EL, Rodebaugh TL, Hassett AL, et al. Psychological sequelae of surgery in a prospective cohort of patients from three intraoperative awareness prevention trials. Anesth Analg 2015;120:87-95.

8 Pandit JJ, Andrade J, Bogod DG, et al. 5Th national audit project (NAP5) on accidental awareness during general anaesthesia: summary of main findings and risk factors. $\mathrm{Br} J$ Anaesth 2014;113:549-59.

9 Avidan MS, Jacobsohn E, Glick D, et al. Prevention of intraoperative awareness in a high-risk surgical population. $N$ Engl J Med 2011;365:591-600.

10 Avidan MS, Zhang L, Burnside BA, et al. Anesthesia awareness and the bispectral index. N Engl J Med 2008;358:1097-108.

11 Zhang C, Xu L, Ma Y-Q, et al. Bispectral index monitoring prevent awareness during total intravenous anesthesia: a prospective, randomized, double-blinded, multi-center controlled trial. Chin Med J 2011;124:3664-9.

12 Mashour GA, Avidan MS. Intraoperative awareness: controversies and non-controversies. Br J Anaesth 2015;115:i20-6.

13 Myles PS, Leslie K, McNeil J, et al. Bispectral index monitoring to prevent awareness during anaesthesia: the $\mathrm{B}-\mathrm{Aw}$ are randomised controlled trial. Lancet 2004;363:1757-63.

14 Avidan MS, Mashour GA. Prevention of intraoperative awareness with explicit recall making sense of the evidence. J American Soc Anesthesiologists 2013;118:449-56.

15 Wunsch $\mathrm{H}$, Linde-Zwirble WT, Angus DC, et al. The epidemiology of mechanical ventilation use in the United States. Crit Care Med 2010;38:1947-53.

16 Easter BD, Fischer C, Fisher J. The use of mechanical ventilation in the ED. Am J Emerg Med 2012;30:1183-8.

17 Kaplan LJ, Bailey H. Bispectral index (bis) monitoring of ICU patients on continuous infusion of sedatives and paralytics reduces sedative drug utilization and cost. Critical Care 2000;4:P190. 
18 Wagner BK, Zavotsky KE, Sweeney JB, et al. Patient recall of therapeutic paralysis in a surgical critical care unit. Pharmacotherapy 1998;18:358-63.

19 Fraser GL, Riker RR. Bispectral index monitoring in the intensive care unit provides more signal than noise. Pharmacotherapy 2005;25:19S-27.

20 Kimball D, Kincaide RC, Ives C, et al. Rapid sequence intubation from the patient's perspective. West J Emerg Med 2011;12:365.
21 Miner JR, Haug E, Friewald S, et al. Evaluation of bispectral EEG analysis and recall in paralyzed intubated patients. Acad Emerg Med 2002;9:405-6.

22 Puller J, Juhasz K, Zerkle S, et al. 24 PRIER: patient recall in emergency rapid sequence intubation. Ann Emerg Med 2017;70:S11.

23 Smith B, Bishop R. Incidence of recall of emergency intubation: a preliminary report. Emerg Med 1998;10:223-5.

24 Finlay T, Parke T. Awareness in the emergency department: a patient's story. J Intensive Care Soc 2016;17:175-7. 